\title{
Secrum microRNA-122 with HCC in chronic hepatitis C
}

\section{Qian Zhang, Yonggui Zhang, Ping Zhao, Jian Jiao, Jiangbin Wang, Wenqian Qi}

\author{
Digestive Department, China-Japan Union Hospital/The third Hospital affiliated to Jilin University, Jilin \\ Province, China
}

Keywords: circulating miRNA, miRNA-122, hepatocellular carcinoma, molecular diagnostics.

\begin{abstract}
To evaluate the correlation of secrum microRNA-122 with hepatocellular carcinoma, serum samples were obtained from 50 patients with hepatocellular carcinoma and 50 age-matched and sex-matched chronic HCV volunteers. miRNAs were isolated from the serum samples. Expression of miRNA-122 in cases and controls was quantified using U6 sn RNA as the internal control. Results show that the serum miRNA-122 level in patients with hepatocellular carcinoma was significantly reduced in comparison with controls and correlated with known risk factors for hepatocellular carcinoma. The serum miRNA-122 of late stage were significantly higher than the one of early stage. HCC patients with low miRNA-122 expression were correlated with lower survival in patients compared with those with higher miRNA-122 expression. Therefore, the serum miRNA-122 level correlates with risk factors for hepatocellular carcinoma. MiRNA-122 may be as a potential diagnostic tool for hepatocellular carcinoma. Further analysis is needed to evaluate the diagnostic power of miRNA-122 for hepatocellular carcinoma.
\end{abstract}

\section{Introduction}

Hepatocellular carcinoma (HCC) may be the third leading causeof cancer-related deaths all over the world, by having an believed 21,000 new cases diagnosed yearly and comprising 700,000 deaths yearly. The incidence of HCC is greatest in regions endemic for hepatitis B virus (HBV) or hepatitis $\mathrm{C}$ virus (HCV). $1 \mathrm{HCC}$ is extremely malignant and deadly, mainly because of the fact that just a part of HCC is operable during the time of diagnosis, when people are already in an advanced stage of disease and therefore possess a poor prognosis.2 Therefore, early recognition of HCC is important for effective treatment and survival. Presently, diagnosing ways of HCC still depends on the radiology, ultrasound along with serum alpha-fetoprotein (AFP). These techniques couldn't avoid numerous false positives and false negtives in patients with increased benign liver disease, for example hepatitis and cirrhosis.3-4 Along with a sensitive and particular early diagnostic marker of HCC may be the great needed. Therefore, identifying novel and noninvasive biomarkers to identify HCC rich in sensitivity and specificity is really a high priority to enhance the prognosis and survival in patients with HCC. In recent reports, dysregulation of miRNAs was discovered to experience critical roles in tumorigenesis and tumor development. MicroRNAs (miRNAs) are roughly 22-nucleotide, noncoding, endogenous RNA molecules with a huge role in many biological processes, including embryonic development, cell differentiation, and tumorigenesis.5. Furthermore, miRNA expression profiles could be unique to malignant tissues, as well as specific to certain kinds of cancer.3In HCC, miRNAs have been located to become aberrantly expressed and a number of them are functionally involved with HCC carcinogenesis, progression, and metastasis $[8,13,14]$ However, the roles of a lot of miRNAs continue to be untouched in HCC. An HCC-specific miRNA expression profile continues to be identified and located to differentiate liver tumors from normal liver tissue rich in precision. 4 Additionally, expression of miRNA-122, a liver-specific and also the most abundant miRNA within the liver, is repressed in HCC.6-8 These decreased miRNA-122 levels are connected having a poor prognosis and metastasis in HCC,9-11 that also plays a role in the malignant phenotype of HCC cells.12Although these data established that downregulation of miR-100 might play a huge role during HCC progression, the clinical value of miR-100 in human HCC continues to be unclear and remains elucidated. 
Within this essay, we'll evaluat the need for miRNA 122a like a market of HCC for recognition of cancer, through investigating miRNA-221 and miRNA-122 expression in serum samples from several patients with HCC and controls matched for age and sex13.

\section{Materials and methods}

Patient information. Fifty patients being treated for HCC from January 2011 to June 2012 were enrolled in the study. In all cases, a pathologist confirmed the clinical diagnosis of HCC. Fifty volunteers matched for age and sex were controls. All patients and volunteers were investigated for family medical history. The study was reviewed and approved by the medical research ethics committee. A questionnaire was used to collect the data from all study subjects, which included age, sex, family history, tobacco smoking, alcohol consumption, and HBV or HCV infection.

Plasma preparation. $2 \mathrm{~mL}$ of peripheral venous blood was collected from all the patients and controls, and stored at room temperature for no longer than two hours before further processing. Blood samples were centrifuged then discarded. The purified plasma samples were transferred to fresh Eppendorf tubes and stored at $-80^{\circ} \mathrm{C}$ for future miRNA extraction.

MiRNA extraction. Total RNA was extracted from plasma using BioZOL ${ }^{\mathrm{TM}}$ total RNA extraction reagent, according to the manufacturer's instructions. The resulting RNA samples were dissolved in $40 \mu \mathrm{L}$ of nuclease-free water and stored at $-80^{\circ} \mathrm{C}$ for future use.

Quantification of plasma miRNA. Expression of serum miRNA-122 was measured using SYBR Green microRNA quantitative reverse transcription polymerase chain reaction reagents (CoWin Biotech Co, Ltd, Beijing, People's Republic of China). The primers used in the quantitative miRNA PCR were as follows: miRNA-122 (5'-TGG AGT GTG ACA ATG GTG TTT GT-3'), and U6snRNA (5'-CAC CAC GTT TAT ACG CCG GTG-3'). Melting curve analysis was performed at the end of the PCR cycles in order to validate the specificity of the expected PCR product. The reaction conditions were as follows: $95^{\circ} \mathrm{C}$ for one minute, 50 cycles at $95^{\circ} \mathrm{C}$ for 15 seconds, and $60^{\circ} \mathrm{C}$ for 30 seconds. The Ct values were determined using SDS2.1 software. The quantitative value for a given sample of miRNA was calculated by subtracting the Ct value of U6 RNA, which served as the internal reference. The relative expression of specific miRNA was determined by the $\Delta \mathrm{Ct}$ value, which was defined as $\Delta \mathrm{Ct}=(\mathrm{Ct}$ miRNA of sample $\mathrm{x}-\mathrm{Ct}$ U6RNA of sample $\mathrm{x})$.

Serum AFP detection. Serum AFP values were determined by AFP chemiluminescence assay using a Beckman i800 luminometer (Beckman Coulter, Chongqing, People's Republic of China). The normal maximum AFP value is $9 \mathrm{ng} / \mathrm{mL}$.

Statistical analysis. The statistical analysis was performed using SPSS version 19.0 software (SPSS Inc, Chicago, IL). MiRNA levels between the cases and controls were determined by the independent samples test. Data on the relative expression of miRNA is shown as the mean \pm standard deviation. Comparison of percentages was done using the Pearson Chi-square test or Fisher's Exact test. Diagnostic values for the different classifiers (miRNAs or AFP) were determined by calculating the area under the receiver operating characteristic (ROC) curve. P, 0.05 was considered to be statistically significant.

Table 1 Clinical and demographic characteristics of patients with hepatocellular carcinoma and healthy controls

\begin{tabular}{lccccc}
\hline Varibles & case $\mathrm{n}=50(\mathrm{n} \%)$ & control $\mathrm{n}=50(\mathrm{n} \%)$ & $\mathrm{P}$ & \\
\hline age & $51.78 \pm 11.9$ & $49.86 \pm 12.9$ & & 0.148 \\
sex male rate(n\%) & 42 & 84 & 41 & 82 & 0.843 \\
$\quad$ HCV RNA positive rate(n\%) & 44 & 88 & 39 & 78 & 0.201 \\
AFP positive rate (n\%) & 44 & 88 & 1 & 2 & $<0.001$ \\
Alt(IU/L) & $78.54 \pm 32.42$ & & $73.41 \pm 38.67$ & & 0.985 \\
AST & $65.72 \pm 21.48$ & $59.87 \pm 23.45$ & & 0.846 \\
TBIL & $63.21 \pm 24.98$ & $57.65 \pm 23.46$ & & 0.765 \\
\hline
\end{tabular}




\section{Results}

\subsection{Patient characteristics}

Fifty HCC patients and 50 volunteers matched for age, sex, and HCV infection served as controls. The AFP level was significantly higher in the HCC group compared with the controls (Table 1).

\subsection{Comparison of Serum miRNA-122 levels in HCC patients and controls}

Compare the MiRNAs from the peripheral venous blood of patients and controls. There was a significantly lower miRNA-122 level in serum samples from HCC patients, $P<0.05$. The univariate Cox regression analyses for miR-122 serum levels revealed a significant association between the miR-122 serum concentration and OS. (Figure 2)

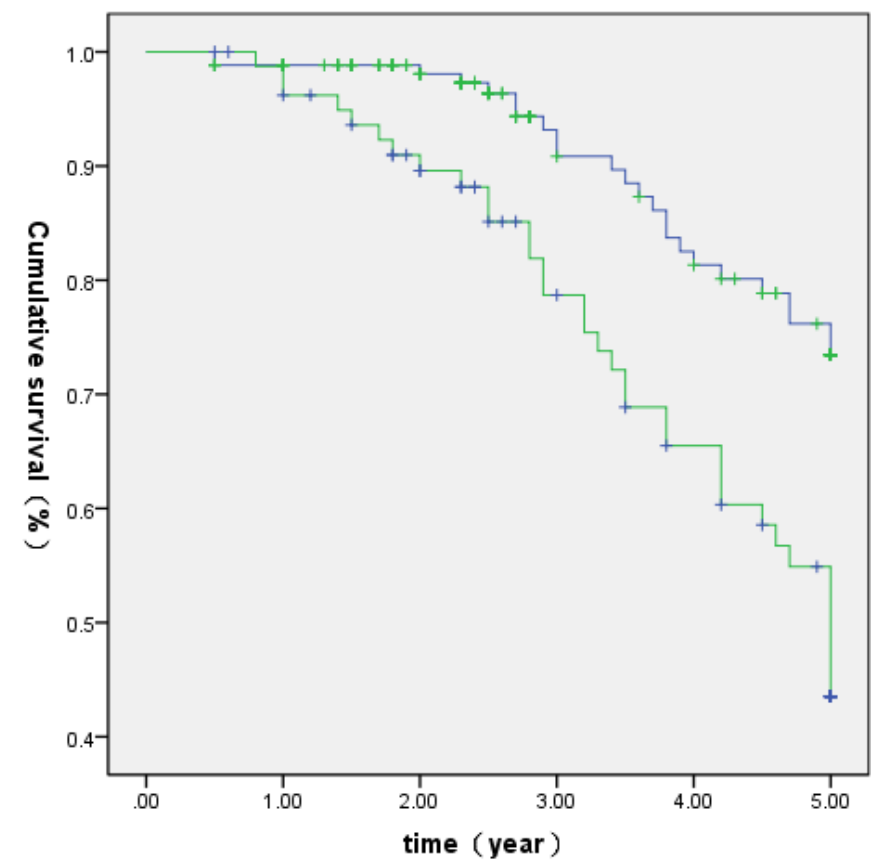

Figure 1 Survival curves for patients with high and low miR-122 serum levels. The P values were calculated with the Cox regression model.

\subsection{Diagnostic value of miRNA-122 and AFP in HCC}

In order to evaluate the prognostic value of miR-122 expression in HCC patients, the detailed clinical information of all HCC patients was reviewed. By means of log-rank test, HCC patients with low miR-122 expression were correlated with lower survival in patients compared with those with higher miR-122 expression. Besides, advanced TNM stage, more Tumor number , and cirrhosis were statistically significant risk factors affecting survival of patients with HCC, whereas others were not of predictive values.

\begin{tabular}{cccc}
\hline Features & OR & $95 \%$ CI & $\mathrm{P}$ \\
\hline Gender & 1.543 & $1.026-1.987$ & $<0.05$ \\
Age & 0.89 & $0.368-01.563$ & $>0.05$ \\
TNM stage & 6.502 & $1.354-18.554$ & $<0.01$ \\
Tumor size & 1.685 & $1.386-4.165$ & $>0.05$ \\
Serum AFP level & 1.612 & $0.700-4.253$ & $>0.05$ \\
Tumor number & 1.508 & $1.432-4.383$ & $<0.05$ \\
cirrhosis & 2.468 & $1.507-60607$ & $<0.05$ \\
miRNA 122 & 20.364 & $2.986-34.457$ & $<0.01$ \\
\hline
\end{tabular}

\subsection{Discussion}

HCC is really a lethal malignancy and it is difficult to cure through the current treatment. Recently, 
miRNAs emerged like a new type of diagnostic tools for identification of cancer. Because miRNA expression profiles are tissue-specific and differ between malignant and normal tissues, these kinds of small RNAs might be able to function as a marker to discriminate tumor tissue from normal tissue or prognostic purposes.21 Although several groups have investigated the function of miRNA like a tumor marker in HCC,14 the majority of the reports used histological examples, and thus have limited clinical significance. Considering that miRNAs are stable within the circulation, we advise that they're going to function as ideal noninvasive biomarkers for proper diagnosis of HCC. Therefore, we examined plasma amounts of two miRNAs in patients with HCC and controls, and evaluated their diagnostic performance poor AFP, the traditional diagnostic marker of HCC.

Expression of miRNA-122 is liver-specific and makes up about about $70 \%$ of total miRNA expression within the liver. Recent reports have shown that miRNA-122 expression is decreased during liver tumorigenesis and miRNA-122 can behave as a tumor suppressor.15-16 In line with these bits of information, we observed a substantial reduction in serum miRNA-122 levels in patients with HCC in contrast to controls. The diagnostic potential of miRNA-122 by calculating AUC values around the ROC curve for serum miRNA-122 and AFP. Interestingly, the AUC of AFP within our study was much greater compared to range formerly reported.17-19

Lanford et al18 discovered that miRNA-122 was involved with controlling viral replication in HCV-related liver cancer, suggesting the miRNA-122 expression level might be connected using the rate of HCV infection. Within our study, info on HCV infection wasn't collected. Unlike western populations, HCV infection isn't a major risk factor for HCC within the People's Republic of China. Rather, HBV infection is really a higher risk factor for HCC within the Chinese population.20,21 Studies from the 3 groups have shown that endogenous miRNA-122 can hinder expression of HBV, suggesting a job for miRNA-122 in HBV-related liver cancer.21-25Within our study, serum miRNA-122 levels in HCV-positive patients were rather lower in comparison to individuals chronic HCC controls. These is comparable along with other study about miRNA 122 with chronic HBV patients.

In conclusion, based on the outcomes of this research, miR-122 is a possible marker for HCCpatients for an additional reasons. The very first is the elevated expression of miR-122 occurs in an initial phase from the multistep procedure for HCC. Second, miR-122 expression is strongly correlated with advanced TNM stage. The 3rd reason is miR-122 may predict the prognosis of patients with HCC.our data claim that the serum miRNA-122 level has some value like a diagnostic tool for HCC.

\section{References}

[1] Curado MP, Edwards B, Shin HR, et al. Cancer Incidence in Five Continents. Lyon, France: International Agency for Research in Cancer Scientific Publications; 2007. Available from: http://www.iarc.fr/en/publications/pdfs-online/epi/sp160/CI5vol9-A.pdf. Accessed April 28, 2013.

[2] Schwartz M, Roayaie S, Konstadoulakis M. Strategies for the management of hepatocellular carcinoma. Nat Clin Pract Oncol. 2007;4: 424-432.

[3] Calin GA, Croce MC. MicroRNA signatures in human cancers. Nat Rev Cancer. 2006; 6: 857-866.

[4] Lu J, Getz G, Miska EA, et al. MicroRNA expression profiles classify human cancers. Nature. 2005; 435: 834-838.

[5] Ladeiro Y, Couchy G, Balabaud C, et al. MicroRNA profiling in hepatocellular tumors is associated with clinical features and oncogene/tumor suppressor gene mutations. Hepatology. 2008; 47: 1955-1963.

[6] Ambros V. The functions of animal microRNAs. Nature. 2004;431: 350-355.

[7] Esquela-Kerscher A, Slack FJ. Oncomirs - microRNAs with a role in cancer. Nat Rev Cancer. 2006; 6: 259-269. 
[8] Fu X, Wang Q, Chen J, et al. Clinical significance of miRNA-221 and its inverse correlation with p27(Kip1) in hepatocellular carcinoma. Mol Biol Rep. 2011; 38: 3029-3035.

[9] Gramantieri L, Fornari F, Ferracin M, et al. MicroRNA-221 targets Bmf in hepatocellular carcinoma and correlates with tumor multifocality. Clin Cancer Res. 2009; 15: 5073-5081.

[10] Galardi S, Mercatelli N, Giorda E, et al. miRNA-221 and miRNA-222 expression affects the proliferation potential of human prostate carcinoma cell lines by targeting p27 Kip1. J Biol Chem. 2007;282: 23716-23724.

[11] Fornari F, Gramantieri L, Ferracin M, et al. MiR-221 controls CDKN1C/p57 and CDKN1B/p27 expression in human hepatocellular carcinoma. Oncogene. 2008; 27: 5651-5661.

[12] Tannapfel A, Grund D, Katalinic A, et al. Decreased expression of p27 protein is associated with advanced tumor stage in hepatocellular carcinoma. Int J Cancer. 2000; 89: 350-355.

[13] Nakai S, Masaki T, Shiratori Y, et al. Expression of p57(KIP2) in hepatocellular carcinoma: relationship between tumor differentiation and patient survival. Int J Oncol. 2002; 20: 769-775.

[14] Kutay H, Bai S, Datta J, et al. Downregulation of miRNA-122 in the rodent and human hepatocellular carcinomas. J Cell Biochem. 2006; 99: 671-678.

[15] Chang J, Nicolas E, Marks D, et al. miRNA-122, a mammalian liver-specific microRNA, is processed from hor mRNA and may downregulate the high affinity cationic amino acid transporter CAT-1. RNA Biol. 2004; 1: 106-113.

[16] Fornari F, Gramantieri L, Giovannini C, et al. MiR-122/cyclin G1 interaction modulates p53 activity and affects doxorubicin sensitivity of human hepatocarcinoma cells. Cancer Res. 2009; 69: 5761-5767.

[17] Tsai WC, Hsu PW, Lai TC, et al. MicroRNA-122, a tumor suppressor microRNA that regulates intrahepatic metastasis of hepatocellular carcinoma. Hepatology. 2008; 49: 1571-1582.

[18] Bai S, Nasser MW, Wang B, et al. MicroRNA-122 inhibits tumorigenic properties of hepatocellular carcinoma cells and sensitizes these cells to Sorafenib. J Biol Chem. 2009; 284: 32015-32027.

[19] Mitchell PS, Parkin RK, Kroh EM, et al. Circulating microRNAs as stable blood-based markers for cancer detection. Proc Natl Acad Sci U S A. 2008; 105: 10513-10518.

[20] American Cancer Society (ACS). Liver Cancer. 2012. Accessed at http://www.cancer.org/acs/groups/cid/documents/webcontent/003114 -pdf.pdf on January 18, 2013.

[21] Garzon R, Calin GA, Croce CM. MicroRNAs in cancer. Annu Rev Med. 2009;60;167-179.

[22] Chen YT, Kitabayashi N, Zhou XK, Fahey TJ 3rd, Scognamiglio T. MicroRNA analysis as a potential diagnostic tool for papillary thyroid carcinoma. Mod Pathol. 2008; 21: 1139-1146.

[23] Taylor DD, Taylor CG. MicroRNA signatures of tumor-derived exosomes as diagnostic biomarkers of ovarian cancer. Gynecol Oncol. 2008; 110:13-21.

[24] Liu R, Zhang C, Hu Z, et al. A five-microRNA signature identified from genome-wide serum microRNA expression profiling serves as a fingerprint for gastric cancer diagnosis. Eur $\mathrm{J}$ Cancer. 2011; 47: 784-791.

[25] Iorio MV, Ferracin M, Liu CG, et al. MicroRNA gene expression deregulation in human breast cancer. Cancer Res. 2005; 65: 7065-7070. 